les qMaZMÁNUSCRITS.RECHERCHE.INVENTION dobeuw Dide

umane $=$ Gluyo $\rightarrow$ Cames - De tremiay à Althe

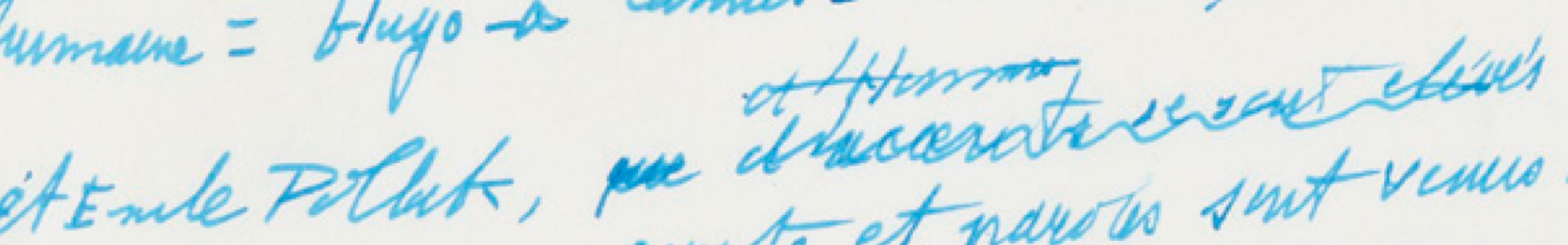
$\frac{39}{14}$ ecuts et parotes suit vemes on

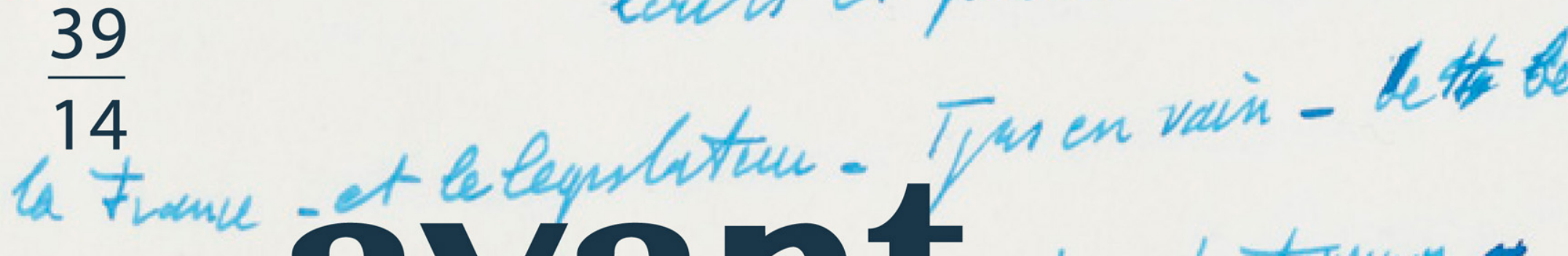

ette Revue

internationale

de critique

génétique

ITEM

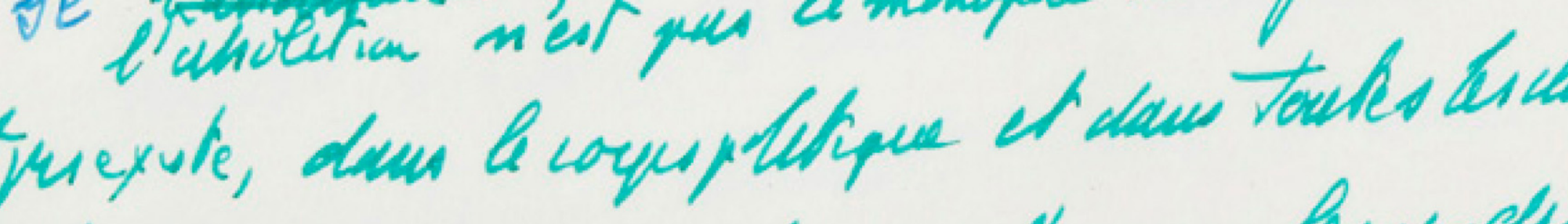

whas, dufenmes et des himes d'queman best de

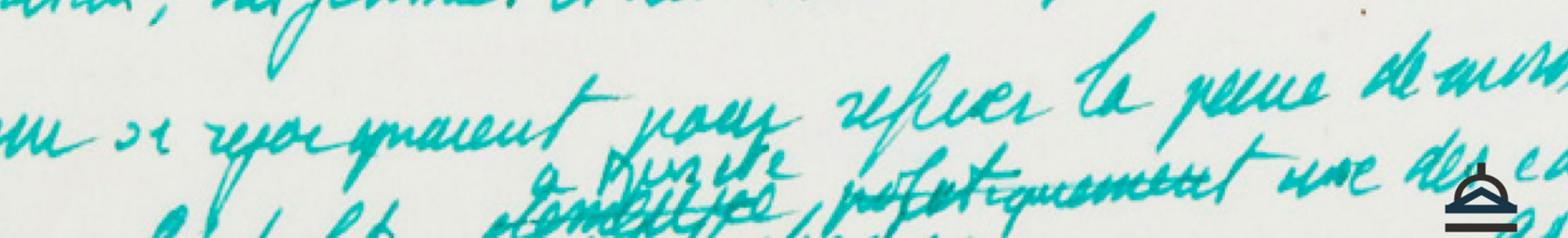

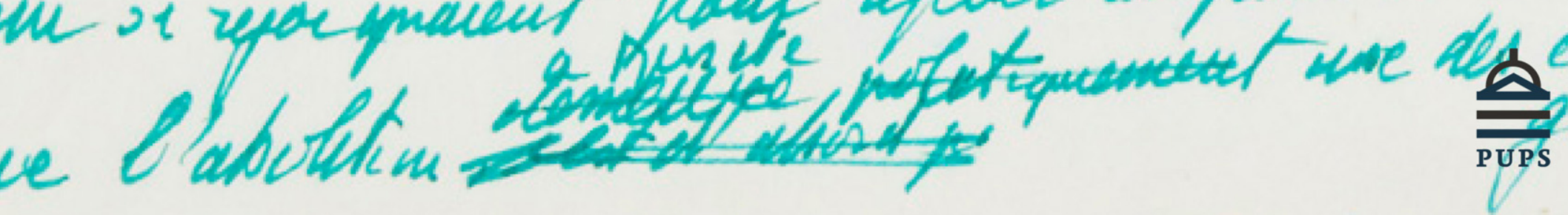




\section{Écrire pour parler. Quelques problématiques premières}

Gilles Philippe

$\mathrm{L}$ e 9 novembre 2012 décédait la seconde femme de T. S. Eliot. On tria les archives, et l'importance de l'œuvre orale du grand poète s'imposa soudain. Des innombrables interventions de circonstance, discours ou conférences, on avait souvent perdu jusqu'au souvenir ; au mieux le lauréat du Nobel en avait-il parfois généreusement confié la publication à des bulletins confidentiels. De ces prises de parole, souvent soigneusement préparées, il restait en tout cas quelques traces : manuscrits et documents préparatoires dont, faute d'enregistrement, la réalisation finale manquait, mais qui se livraient à l'étonnement du spécialiste. De l'œuvre immense du poète et du penseur, nous ne connaissions donc que la partie émergée, celle qu'il avait fait paraître dans les pages du Criterion ou en volume, au long des années, chez Faber and Faber. On se promit alors d'ouvrir le grand chantier des « textes dits », ce qui exigeait une réflexion préalable sur la nature et le statut de leurs « avant-dire », notes ou propos saisis à un stade graphique qu'ils n'étaient pas appelés à conserver. Ce sont quelques-uns des problèmes qui se posent dans ce genre d'entreprise que l'on entend ici envisager.

Cet étrange objet qu'est le texte écrit pour être dit embarrasse, ne serait-ce que parce que la pièce principale manque pour son analyse : la prise de parole effective, livrée sous les contraintes de l'instant et d'un public dont la présence, fût-elle muette, n'en est pas moins agissante, toutes choses dont même l'enregistrement le plus fidèle ne saurait rendre compte. Matériellement, l'avant-dire prend en outre rarement une forme achevée se prêtant à la lecture continue : ce sont fréquemment des suites de mots qui peinent à faire texte ; on ne sait pas toujours ce que l'orateur en a ou en eût fait, ce qu'il en a ou en eût gardé, développé ou élagué ; on mesure aussi et surtout la perte de la parole vive. Mais le document attire notre attention sur ce que nous avions longtemps négligé : que les genres oraux ont aussi une genèse et que cette genèse fut nécessairement ou généralement écrite. Le plaideur ou l'orateur, le conférencier ou le professeur ont le plus souvent des notes ou un texte sous les yeux, qu'ils suivront ou ne suivront pas, mais qu'ils devront transformer en tout autre chose.

Notre négligence étonne d'autant plus que les manuscrits de discours sont des objets de choix pour la patrimonialisation, plus encore que les manuscrits d'œuvres écrites, car
Des corpus instables 
ils gardent trace d'un événement collectif, parfois profondément inscrit dans la mémoire commune. En 2006, la Bibliothèque nationale de France fit ainsi une abondante publicité autour du don du manuscrit final du « Discours pour l'abolition de la peine de mort » de Robert Badinter ${ }^{1}$ (fig. 1) ; commencée en août 1981, une première version avait fait l'objet d'un travail continu jusqu'au moment où le texte fut déclamé à l'Assemblée nationale, le 17 septembre. L'État a en outre souvent préempté pour les Archives nationales des brouillons de discours majeurs, comme, tout récemment, ceux que Robespierre prononça entre 1791 et 1794 (fig. 2).

L'importance historique de certains de ces discours explique par ailleurs que leur genèse, interne ou externe, ait pu passionner le grand public, au point que cette genèse soit ellemême patrimonialisée. On a ainsi célébré à la fin de l'été 2013 le cinquantième anniversaire du discours que Martin Luther King prononça à Washington le 28 août 1963. Son histoire passionna : le texte en avait été préparé avec l'aide de plusieurs conseillers, mais rédigé quelques heures seulement avant d'être délivré. La chose est désormais sûre : la version écrite que King avait sous les yeux ne contenait pas la péroraison qui l'a rendu célèbre, avec l'anaphore du «I have a dream» : un de ses proches lui avait en effet conseillé de ne pas utiliser une nouvelle fois une formule qui avait un goût de cliché et dont l'orateur avait déjà abondamment usé lors d'interventions précédentes. Ce n'est qu'au podium, à la suggestion d'une personne de l'assistance et sous l'inspiration du moment, que l'orateur décida de la reprendre en un spectaculaire geste amplificatoire : jusque-là, semble-t-il, il s'en était plus ou moins tenu au texte qu'il avait au pupitre (un dactylogramme largement amendé, désormais en main privée), la foule écoutait avec admiration mais sans enthousiasme notable. La partie la plus célèbre du discours n'eut donc pas de genèse écrite ; elle fut en quelque sorte appelée par un subit changement de ton et de posture oratoire, King quittant soudain son allure de militant des droits civiques pour adopter celle d'un prêcheur baptiste ${ }^{2}$.

De nombreux autres exemples mériteraient ici mention, et l'on se contentera de rappeler le cas d'un autre discours majeur de l'histoire politique américaine, car il fait encore l'objet de débats nourris entre spécialistes. Inspirées d'un discours de Périclès, les dix phrases (soit environ deux minutes de parole) de l'« Adresse » prononcée à Gettysburg par Abraham Lincoln le 19 novembre 1863 (tout Américain en connait les premiers mots, ils sont gravés dans le marbre du Mémorial de Washington : "Four scores and seven years ago... ») n'avaient guère été conçues et ne furent guère prononcées que comme un bref complément prenant acte du discours de deux heures proclamé par le grand orateur du temps, le sénateur Edward Everett. Le statut exact des cinq manuscrits autographes de l'Adresse a donné lieu à des spéculations sans fin : deux seulement semblent bien avoir précédé la version orale, quoiqu'on ne sache point ce que Lincoln avait sous les yeux ni ce qu'il dit précisément.

Le cas le mieux connu en France demeure sans aucun doute celui de l'Appel du 18 juin du général de Gaulle, seul discours (et seul manuscrit de discours) retenu à ce jour par le programme « Mémoire du monde » de l'Unesco. Comme l'Adresse de Gettysburg, l'Appel a connu une genèse " continuée », dont les problématiques croisent, en bien des

1. Il est désormais en ligne sur Gallica : <http://gallica.bnf.fr/ark:/12148/btv1b8571107s>.

2. Nous sommes désormais bien informés sur la genèse de ce discours grâce à l'ouvrage de Gary Younge,

The Speech. The Story behind Dr. Martin Luther King Jr's Dream, Chicago, Haymarket Books, 2013. 
Ent comun upendant, a tyocorepues, - ectec.

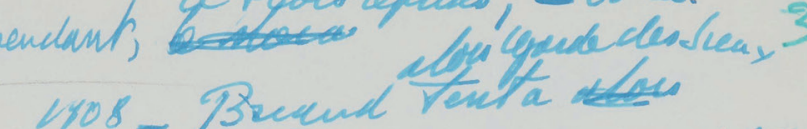

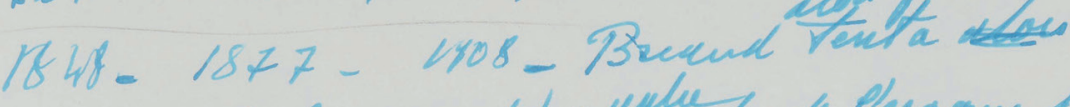

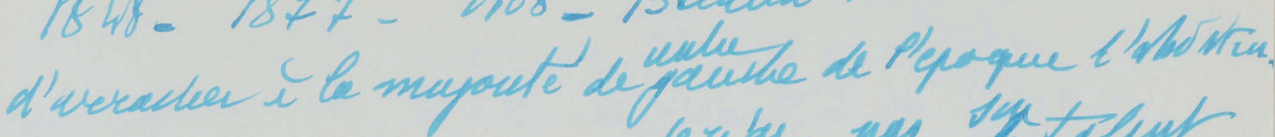

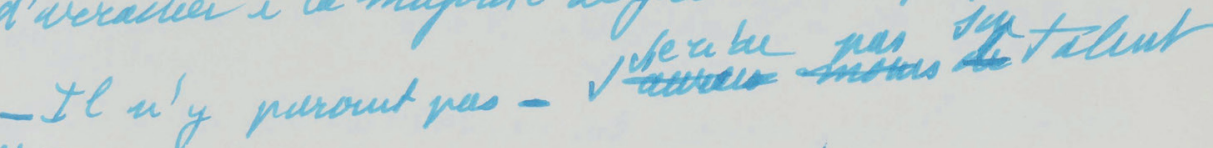
Mis

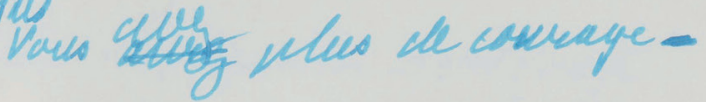

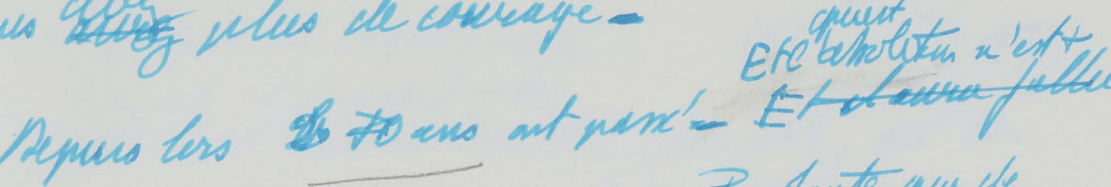
cltte jamais vemue pusqu la vos. Tas faute que de

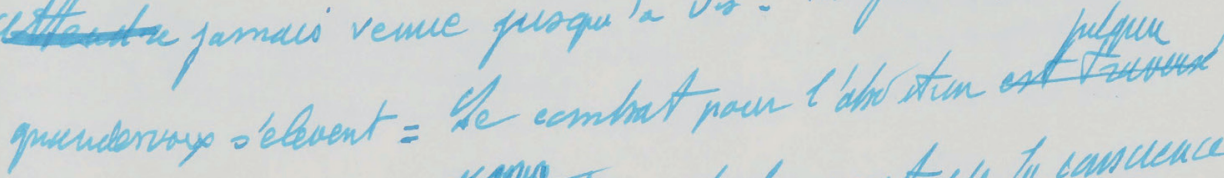

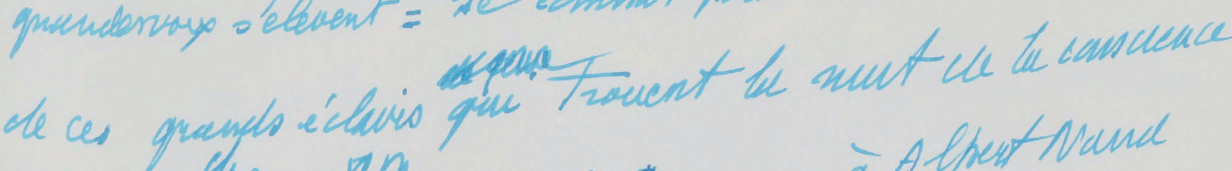
Lumare = Hugo as Cames - De tremcoux à Alpent Nand

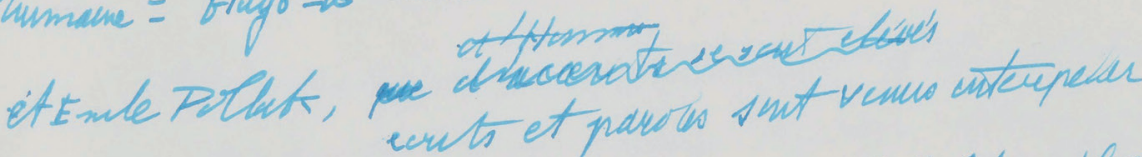

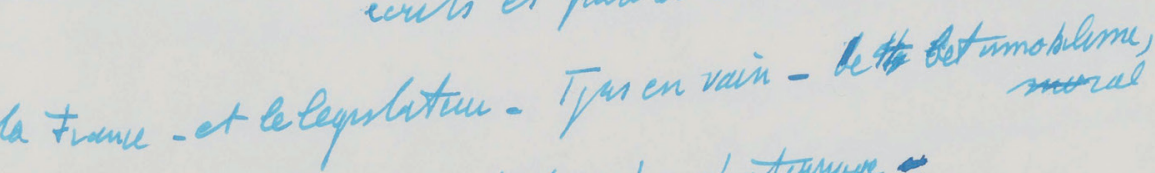

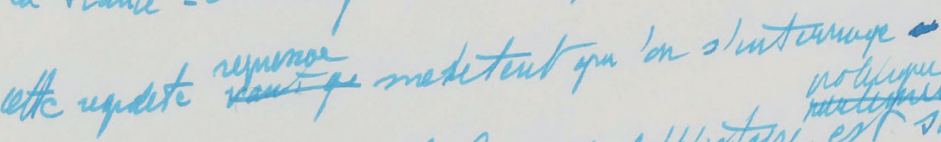

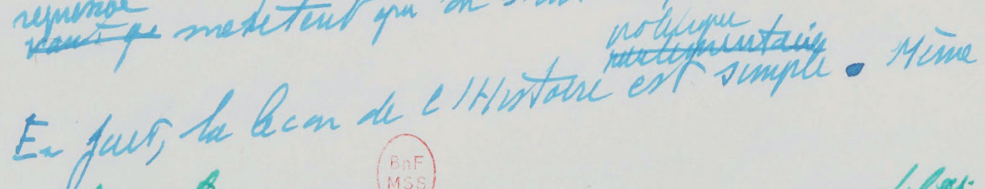

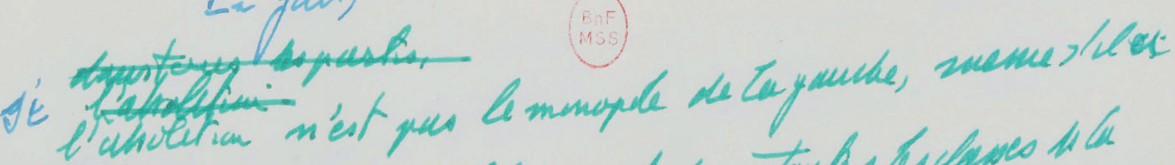

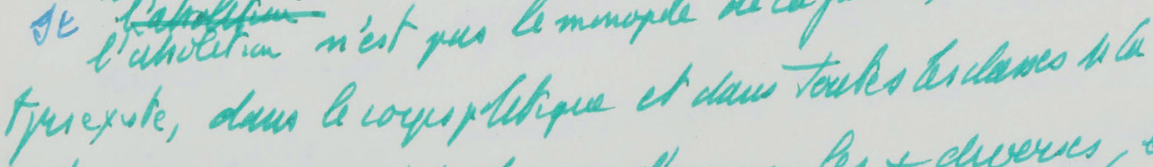
netian, def fermes et des himes diqueman bert deverses, of

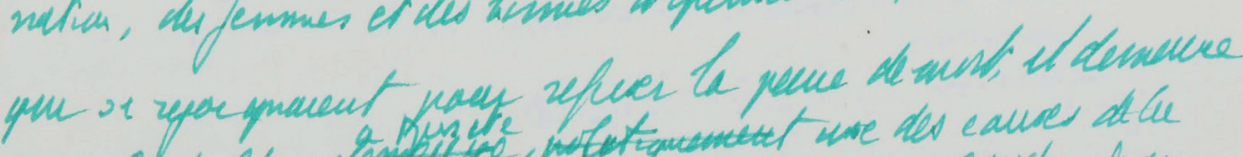
que

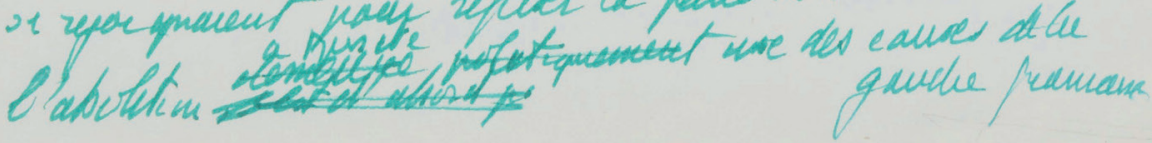

Fig. 1 : Robert Badinter, Manuscrit du discours pour l'abolition de la peine de mort prononcé le 17 septembre 1981 à l'Assemblée nationale (première version, août 1981) (BnF, NAF 28085) 
points, les processus de genèse post-éditoriale qui intéressent aujourd'hui fortement les spécialistes de génétique de l'écrit ${ }^{3}$. On sait qu'aucun enregistrement de la version diffusée par la BBC n'a été conservé et que son texte ne nous est connu que par la transcription des services d'écoute suisses. Il est cependant acquis qu'à la demande du Foreign Office, de Gaulle semble avoir modifié tardivement l'exorde de l'Appel lancé à la radio, qui différait au moins sur ce point du « texte officiel » postérieur. Outre une version manuscrite subsistent plusieurs versions publiées, et l'on sait par ailleurs que le texte de l'Appel fut décliné par de Gaulle dans d'autres messages radiodiffusés à la fin de juin 19404. On est assurément ici face à un cas assez banal d'établissement de texte, à ceci près que le choix de la « bonne version » n'est pas réglé par la simple décision de l'auteur d'entériner et de publier une version définitive, puisque celle-ci n'efface pas l'événement même de la première publication orale. De plus, si l'on veut bien admettre que l'enregistrement assure à la performance orale une reproductibilité comparable à celle du texte écrit, le passage d'une version orale à une version écrite d'un même discours, celles-ci fussent-elles rigoureusement identiques, efface tous les choix ou habitus suprasegmentaux de l'orateur (ton, prosodie, accentuation...).

Dès lors, le protocole menant à la sélection de la «version définitive » d'un discours oral ne saurait s'établir sur une base aussi simple que celui qui préside généralement à celle de la « version définitive » d'un texte écrit, à savoir le dernier état publié à l'initiative ou avec l'accord de l'auteur. Albert Camus, par exemple, a bien « validé » une version écrite de la conférence sur «L'artiste et son temps », prononcée à Uppsala le 14 décembre 1957, peu après son acceptation du prix Nobel : il l'a en effet fait paraître en février 1958 chez Gallimard dans le volume contenant les deux Discours de Suède. Mais il avait prononcé quatre fois cette conférence, en 1954 et $1955^{5}$, et il n'est pas sûr qu'entre ces différentes versions orales, le texte en eût-il été identique, il eût préféré en retenir la dernière comme la meilleure. Le corps et le public, l'heure et le lieu entrant en matière, l'ultime performance n'était peut-être pas supérieure aux premières. Plus encore que le suprasegmental, la circonstance ignore le ne varietur ; la parole est sans itérabilité.

L'avant-dire : formes et points de fuite
Les notes prises en vue d'une intervention orale ont le plus souvent un statut fonctionnel fort différent des manuscrits prérédactionnels destinés à une réalisation finale écrite : elles ne sont pas nécessairement, ou pas à proprement parler, une première version du texte qui sera prononcé et consignent parfois des informations plus que des formulations. Nombre d'avant-dire témoignent ainsi du souci de trouver une juste mesure entre la nécessité d'avoir un texte suffisamment établi pour que le propos soit ferme, et suffisamment lâche pour que l'orateur puisse respecter la convention d'une prose dite et non lue.

3. C'est en effet une question dont s'est emparée l'équipe « Manuscrit, Linguistique, Cognition » de l'ITEM (CNRS-ENS), sous l'impulsion de Rudolf Mahrer.

4. Voir Thierry Herman, Au fil des discours. La rhétorique de Charles de Gaulle (1940-1945), Limoges, LambertLucas, 2008.

5. Voir la Note sur le texte de Raymond Gay-Crosier et Maurice Weyembergh dans Albert Camus, Euvres complètes, t. IV, Paris, Gallimard, coll. « Bibliothèque de la Pléiade », 2008, p. 1400. 


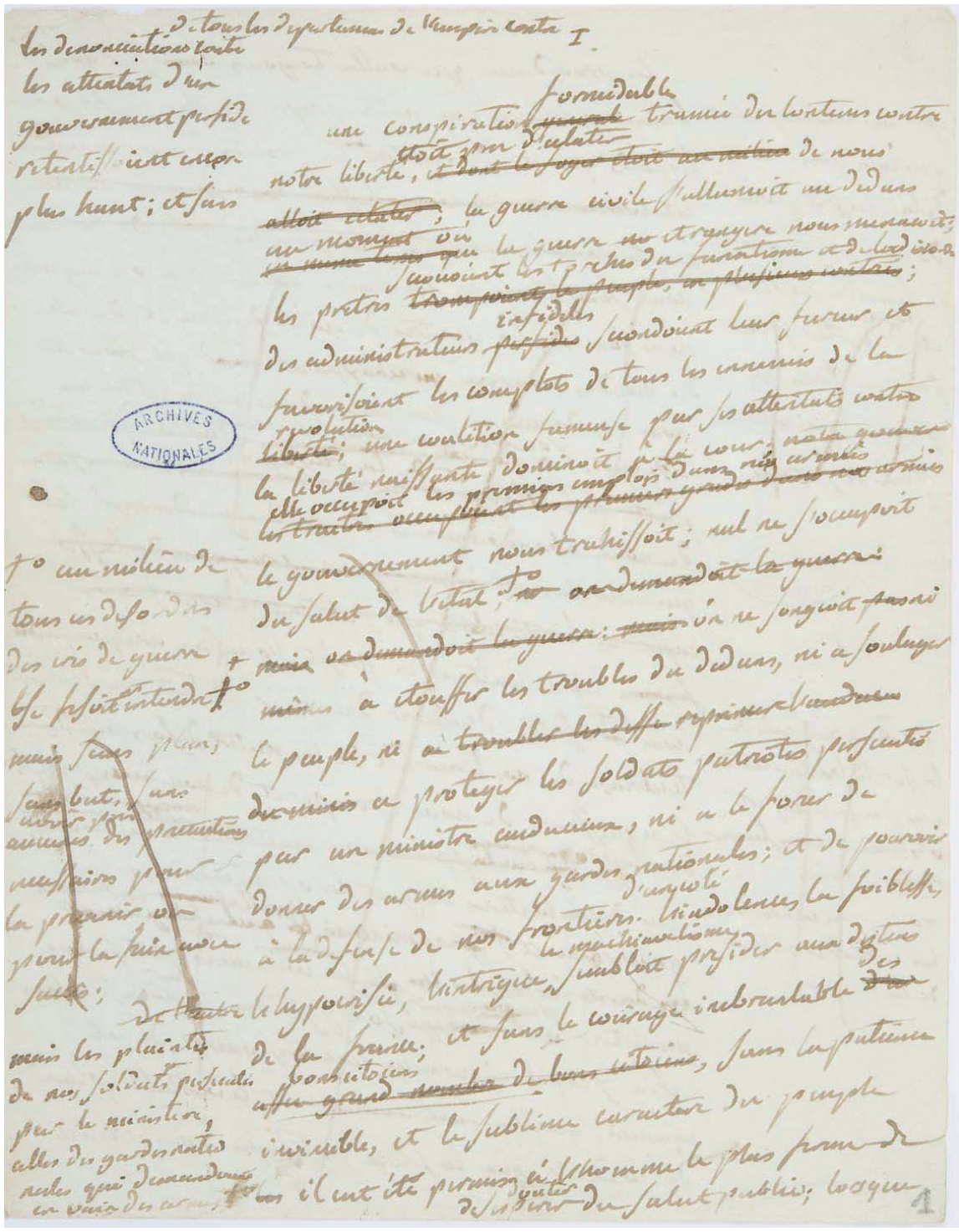

Fig. 2 : « [Robespierre se résigne à la guerre.] Discours aux Jacobins sur les circonstances actuelles », 26 mars 1792, f. 1 (Document conservé aux Archives nationales, Pierrefitte-sur-Seine)

Une technique courante consiste à ne rédiger que partiellement le texte de la conférence. C'est ce que fit par exemple Albert Camus pour une intervention qu'il donna devant de jeunes confrères ou futurs confrères journalistes ; la date et la circonstance exactes ne nous sont pas connues, mais un manuscrit nous est parvenu, dont il y a tout lieu de penser que c'est bien celui que Camus avait sous les yeux au moment de prendre la parole. L'introduction et la conclusion y apparaissent entièrement rédigées, avec un évident travail de l'exorde («Vous allez être ou vous êtes, Messieurs, journalistes 6 ») et une péroraison qui appelle la grandiloquence («De ce point de vue, le journalisme aujourd'hui peut être ou bien rien ou bien une grande aventure. Mais j'ai une trop haute idée de la jeunesse de mon pays pour douter un seul moment que la plupart d'entre 
vous n'accepte de la courir, pour l'honneur de ce que nous sommes $\left.{ }^{7} »\right)$. La rédaction inaugurale se déroule jusqu'à la phrase qui annonce l'entrée dans le vif du sujet; le corps du développement n'est plus alors qu'un simple plan, essentiellement constitué de phrases nominales sous-ponctuées présentant une suite de thèmes sur lesquels l'orateur se sait ou se veut capable d'improviser. Bien qu'il s'agisse ici de l'essentiel de la conférence, ces notes sont bien moins longues que la conclusion et deux fois plus brèves que l'introduction. Elles prennent la forme suivante :

Coup d'œil sur la presse d'aujourd'hui

Technique de Paris-Soir

Technique de l'argent. Le statut de la presse

Technique de l'insulte 8

Cet exemple nous met face au paradoxe foncier de l'avant-dire : ce document n'est pas appelé à être vu par autrui, et certains de ses segments marquent un net décrochage des opérations communicationnelles formelles, alors même que les passages rédigés sont très fréquemment saturés de marques interpellatives et déictiques et que l'ensemble vise, dès ce stade, à créer un discours adressé.

La chose s'observe fort bien dans de nombreux avant-dire, même si les configurations manuscrites peuvent être très différentes. Celle que présentent les archives du discours prononcé par Albert Camus devant un parterre de confrères et apprentis confrères n'est peut-être pas la mieux représentée, et sans doute sera-t-on mieux éclairé si l'on regarde sous cet angle la transcription de notes prises par le tout jeune Jean-Paul Sartre en vue d'une conférence sur John Dos Passos donnée au Havre en février $1933^{9}$ :

III

Les sujets personnages

Se moque bien de la question des réalités collec tives : individu et rapports interindividuels ou groupes existant réellement.

Mais pour lui : il y a bien peu de choses individuelles dans un homme : tous les mêmes organismes - élevés différemment suivant les classes - font tous des gestes stéréotypés et dictés par leur condition sociale, leur éducation et les événements.

Progrès sur Dreiser

Pourtant : vus du dedans

Représentent des points de vue : 10 personnages

10 points de vue qui alternent.

Coupure nette de classes

7. Ibid., p. 1339.

8. Ibid., p. 1337.

9. On trouvera de ce texte une version transcrite par Isabelle Grell, révisée et annotée par Julien Piat, dans A. CohenSolal et G. Philippe (dir.), Études sartriennes, n 16, « Les conférences du Havre sur le roman », 2012, p. 151-154. Les lignes blanches marquent ici le changement de feuillet. Le manuscrit est en main privée. 
\begin{tabular}{l|l|l} 
Les ouvriers & Joe & aventuriers
\end{tabular}

$\mathrm{Mac}$

Charly : l'ouvrier sans opinion définie

Ben Compton : le révolutionnaire

Les petits bourgeois qui optent pour les grands bourgeois

Janey, sœur de Joe, amoureuse du grand Patron

Moorehouse, ses efforts vers la distinction

Les grands bourgeois :

Evelyne

Eleanor

Richard

Moorehouse

Le monde est vu successivement du point de vue de chacun. Le style change pour chacun.

Pour Mac : argot américain - pas d'adjec

tifs.

Pour Eleanor, petite-bourgeoise qui se haussera lentement jusqu'à la grande bourgeoisie, profusion d'adjectifs de couleur, style mièvre, gracieux.

« Ce fut Miss Oléphant qui conseilla à Eleanor de suivre des cours à l'institut d'Art. Elle avait chez elle des reproductions de Bossetti et de Burne Jones et parla à Eleanor des préraphaélites et lui fit sentir que l'art était quelque chose d'un blanc ivoire, et très pur, et noble, et distant, et triste, juste comme elle le désirait. »

$$
\text { 42ème Parallèle } 205
$$

Grâce à ce procédé, ses personnages, étant vus du dedans et dans la mesure où ils se prennent pour des individus, leur individualité est sauvegardée.

Voyez la présentation d'Eleanor.*10

Ainsi tout est décrit par rapport à l'individu. Dans chaque section un indivi du sert de centre provisoire.

Objectivité absolue de Dos Passos. Ne juge jamais. Montre le personnage se jugeant et décrit sans donner son avis.

10. L'astérisque indique que Sartre lit à ce moment de la conférence un passage de John Dos Passos traduit à cette intention par Simone de Beauvoir ; les feuillets manuscrits de cette dernière sont insérés parmi ceux de Sartre. 
Ex. : Moorehouse qu'il déteste, futur grand bourgeois dont l'ascension se fait au prix de petites lâchetés, de petits marchandages, de coquineries sans envergure. Jamais un jugement.

Découvre qu'Annabella, sa fiancée, se donne à n'importe qui. Cependant l'épouse, pour son argent.

Voici comment la chose est présentée.*

Mais d'autre part, les individus qui se voient et se pensent comme individus, qui considèrent leur vie comme une aventure individuelle et qui regardent le monde de leur point de vue indi viduels; ils ne sont pas des individus, càd une substance unique, incomparable. Ils sont tirés à des milliers et des milliers d'exem plaires et ce sont de véritables automates que leur milieu assujettit à un comportement rigoureux.

Dos Passos glisse même sur ce qui est bien à eux, sur leur douleur, p. ex. en ce qu'elle a d'unique, pour insister au contraire sur le typique, l'universel enfance de Mae : voyage de Mae Janey : mort d'Alie - vie de Janey*

De même les débuts d'Eleanor Chicago dans le monde artiste et snob de New York.*

Naturellement il leur arrive des événe ments singuliers. Mais ces événements n’ont rien de l'aventure irréparable et fatale du roman classique. Ce sont de petites rencontres sans conséquences, ou bien qui longtemps après leur permettent de s'élever un peu ou provoquent une déchéance partielle. Puis ils retombent et remontent etc. et chacun de ces événe ments est, dans son individualité même, tout à fait banal, tout à fait typique.

Voyez la conversation de Janey la dactylo qui vise à la distinction bourgeoise avec son frère Joe, qui court le monde - un matelot.*

Mille conversations semblables.

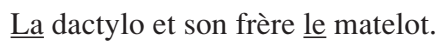

Donc 


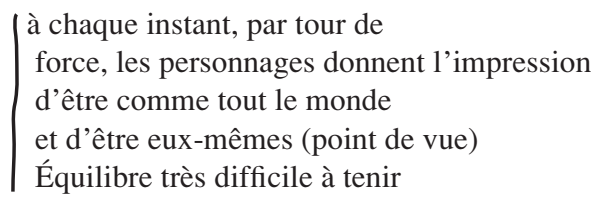

Il semble ainsi que, dans la plupart des cas, l'avant-dire manuscrit alterne des segments rédigés et des segments notationnels. Les premiers ont vocation à être dits tels qu'ils ont été notés (ce ne sont pourtant pas toujours des « formules ») ; les seconds ne reçoivent pas à ce stade leur formulation définitive ; ils préjugent simplement qu'un point doit être abordé en cours de parole. Les premiers respectent donc les grandes règles nécessaires à un bon fonctionnement communicationnel ; les seconds les ignorent :

- prédications binaires (avec thème et prédicat : «Le style change pour chacun »), vs réduction de l'énoncé à son seul thème ou à son seul prédicat (« Progrès sur Dreiser », « Ne juge jamais »);

- distribution de l'information sur plusieurs plans grammaticaux à l'aide, notamment, de la subordination ou de l'apposition (« ce sont de véritables automates que leur milieu assujettit à un comportement rigoureux »), vs suite de termes juxtaposés, le plus souvent des groupes nominaux («Les grands bourgeois : / Evelyne / Eleanor / Richard / Moorehouse »);

- textualisation forte exigée et assurée par les procédures habituelles de rédaction (anaphores, marques de connexité : « mais », « ainsi », «pourtant», « naturellement», « d'autre part »), ainsi que par le recours à des modèles d'agencement textuel intégrés par le locuteur (disposition argumentative, narrative ou expositive des informations, etc.), vs sérialisation sur le mode de la liste («Janey : mort d'Alie - vie de Janey »);

- validation explicite d'un protocole communicationnel (adresse et interpellation, marques de connivence, questions rhétoriques, etc. : "Voici comment la chose est présentée »), attribution d'une force illocutoire et possible recours à des modalités non assertives («Voyez la présentation d'Eleanor»), vs absence de toute référence à la situation oratoire programmée, la note étant rédigée « pour soi-même » (les soulignements sont par exemple destinés à l'orateur : ils peuvent servir de point de repère dans le parcours de la page, indiquer un point de la démonstration à ne pas omettre ou à développer le moment venu, voire signaler qu'un mot doit être accentué : «La dactylo et son frère le matelot »).

Tout cela nous est fort familier puisque, mutatis mutandis, les avant-dire présentent bien souvent la même polarité entre segments rédigés et segments notationnels que les avanttextes proprement dits, à cette différence près que les brouillons de l'écrit se répartissent peut-être plus nettement entre ceux qui relèvent d'une phase prérédactionnelle et ceux qui ressortissent à une phase rédactionnelle. Parce qu'ils ont généralement une genèse plus brève qui ne connaît le plus souvent qu'une seule campagne d'écriture (l'atteste l'extrême difficulté de lecture du manuscrit de Camus que je citais11), les brouillons de l'oral proposent en outre moins de degrés intermédiaires entre segments rédigés et segments non rédigés que les brouillons de l'écrit. Du moins est-ce vrai pour certains auteurs, ce type de

11. Voir A. Camus, Euvres complètes, t. IV, op. cit., p. 1574. Cette remarque ne vaut bien sûr pas pour tous les orateurs et pour les discours les plus formels, mais elle s'observe sans peine dans les manuscrits des auteurs que j'évoque ici. 
comparaison n'ayant guère de pertinence que par rapport aux pratiques avant-textuelles d'un même scripteur à une même époque de sa carrière.

Qui a pu consulter le manuscrit ou la version récemment publiée des conférences sur «La technique du roman et les grands courants de la pensée contemporaine » que JeanPaul Sartre a prononcées entre novembre 1932 et mars 1933 pourra cependant noter que la plupart des avant-dire de ces interventions havraises ne présentent pas l'hétérogénéité syntaxique et linguistique des notes sur Dos Passos. La toute première (elle reste à ce jour inédite), consacrée à un parcours général de l'histoire du roman, présente fort peu de phrases rédigées ou partiellement rédigées et beaucoup de listes; le caractère notulaire de la syntaxe y est encore renforcé par l'absence fréquente d'articles devant les groupes nominaux ; mais ces notes restent formellement proches de celles de la conférence sur Dos Passos. Dès la deuxième conférence, ce protocole devient rare. Dans l'introduction, Sartre omet bien encore quelques mots grammaticaux :

Jusque là puisé dans le fonds

commun des romans contemporains de Mauriac

à Conrad.

Opinions qui appartiennent à tout le monde

et à personne, de notre époque.

Point de départ seulement.

À présent nous allons étudier quelques techni

ques particulières. Voir si elles enrichiront ce fonds commun

de quelques nouveautés 12 .

mais, au bout de quelques feuillets, il préfère rédiger le texte a priori exactement comme il entend le dire :

À cette distinction entre roman et récit

Fernandez oppose la sienne qui fait du récit, nous

l'avons vu, une histoire au passé et du roman

une histoire au présent mais je ne suis pas sûr

du tout que ces deux définitions du roman ne

puissent se ramener l'une à l'autre, quoique, comme

nous le verrons te un [un mot biffé] des graves défauts des F.M sera

précisément la façon dont le temps passe dans ce roman 13 .

S'il est certain que le niveau de rédaction de ces notes dépend largement de facteurs conjoncturels (selon les semaines, Sartre dispose de plus ou moins de temps pour préparer sa conférence ; il est possible qu'il pensât ne pas avoir le temps de prononcer la dernière de la série, celle qu'il entendait consacrer à Dos Passos, etc.), il apparaît en tout cas que le jeune Sartre préfère rédiger autant qu'il lui est loisible, et cela même pour des passages très simples qui se prêteraient fort bien à l'improvisation et présentent pourtant un niveau de «prêt-à-dire » aussi grand que des passages plus complexes. On le notera par exemple en lisant l'introduction de la conférence sur le monologue intérieur :

12. Transcription d'Alexis Chabot et Vincent de Coorebyter (A. Cohen-Solal et G. Philippe (dir.), op. cit., p. 35).

13. Ibid., p. 39. «F.M » désigne Les Faux-Monnayeurs d'André Gide. 
Nous allons étudier aujourd'hui l'évolution d'un procédé technique particulier : le monologue intérieur. Ce travail est assez agréable et facile parce que nous savons exactement quand cette forme d'exposi tion a pris naissance : c'est en 1887 et nous connais sons exactement aussi les lignes d'influence qui conduisent de Dujardin à Joyce et de Joyce à Larbaud et à V. Woolf.

Je me propose donc de montrer comment le monolo gue intérieur, produit des théories littéraires et philoso phiques du Symbolisme finit par servir les fins, après de nombreuses transformations, d'un réalisme absolu, comment, issu d'un courant d'idées nettement idéaliste il passe aux mains de néo-réalistes anglais, comment il se stylise, s'enrichit et, d'abord strictement limité

à nous découvrir une conscience, finit par intégrer $\underline{\text { l'univers entier }}^{14}$

Si l'on compare l'incomparable et que l'on rapporte des avant-dire de conférences comme ceux de Sartre et de Camus aux supports écrits préparés par des avocats à la veille de plaidoiries, on ne s'étonnera cependant guère de trouver dans ces derniers la même double pratique :

Il s'agit parfois de quelques mots, destinés à servir de support au discours largement improvisé qui sera tenu à l'audience. Dans d'autres cas, l'élaboration du texte est très poussée et présente quasiment le caractère d'une plaidoirie achevée, ce qui ne signifie pas pour autant que l'orateur a suivi systématiquement ses notes 15 .

Entre les deux extrêmes, simples notes ou texte pleinement rédigé (choix largement contraint évidemment par l'enjeu judiciaire ou médiatique de la plaidoirie), s'étage toute la gamme des possibles, avec par exemple la juxtaposition de groupes nominaux en attente de formulation et de fragments prétextualisés déjà conçus pour une situation d'adresse orale :

C'est là en effet une première observation

Nous sommes en police correctionnelle : Délits

Domaine civil : actes tout seuls peuvent engendrer conséquences juridiques

Ici élément de plus : culpabilité de l'argent

Comment prévention fera-t-elle la preuve?

D'une part intérêt

D'autre part simulation et mensonges

Spécialement vastes entreprises : insuffisance des écritures, faux bilans, inventaires frauduleux

Tant d'affaires célèbres. Pourtant civiles

14. Transcription de Frédéric Martin-Achard, Études sartriennes, nº 16, p. 76.

15. Yves Ozanam, Les Grandes Plaidoiries. Archives et documents pour l' histoire, de l'affaire Calas au procès de Pétain, Paris, La Martinière, 2011, p. 6. 
Ici délits : mauvaise foi plus grande, cupidité plus éhontée ?

Pas du tout : comptabilité rigoureusement exacte : probité absolue 16

Les soulignements sont en rouge dans le document original : à la relecture le rédacteur semble avoir voulu insister sur l'opposition binaire qui doit fonder sa démonstration. De fait, bien qu'aucune synthèse n'existe encore sur la question, un simple parcours des avant-dire des plaidoiries et des avant-dire des conférences, quand les uns et les autres sont disponibles, semble valider l'intuition d'une pratique possiblement différente dans la disposition matérielle des notes. Celles du plaideur prévoient ce que doit être la performance oratoire : les soulignements à traits simples, doubles ou triples, souvent en couleur, y sont plus nombreux, et la disposition générale des notes sur la page témoigne que le document a une valeur fonctionnelle plus grande. L'avant-dire anticipe le projet oratoire, la rhétorique de l'argumentation l'emportant ici largement sur celle de l'exposition.

Écrire pour parler, parler pour écrire, écrire pour parler puis écrire
L'œuvre d'Albert Camus permettrait à elle seule d'attester que l'étude de l'interaction écrit/oral ne se limite pas, dans les processus de genèse, à la description des manuscrits d'interventions publiques ou à leur comparaison avec la version effectivement prononcée, quand un enregistrement subsiste. Il n'y a somme toute que peu de relations entre les quelques notes que l'auteur put prendre à la dernière minute, sur des supports de fortune, en préparation d'interviews à venir et qu'il craint délicates, et le discours, longuement médité et pleinement abouti, rédigé en vue de la réception du prix Nobel, discours pris dans un double processus de genèse, puisqu'il est d'emblée acquis qu'il doit être publié un jour sous forme écrite ${ }^{17}$. Sans même prendre en considération la représentation de l'oral spontané ou formel dans les textes romanesques (dialogues divers, sermon du père Paneloux dans La Peste, etc.) ou le cas particulier du texte théâtral, on notera qu'un texte de fiction comme La Chute (1956) entretient des rapports complexes avec l'oral : l'histoire du monologue de Jean-Baptiste Clamence, un ténor du barreau déchu, ne s'arrête pas au livre publié en 1956 ; en juillet 1958, Camus en enregistra, à une fin que nous ne connaissons pas, une version " dite ». Les bandes conservées à la Méjanes d'Aix-en-Provence témoignent que Camus ne voulut point tant donner une lecture de son texte qu'en ajuster le « ton » (cette notion cruelle, sur laquelle une réelle synthèse fait encore défaut et qu'il faut appeler de nos vœux), afin de désambiguïser le caractère cynique et délirant du propos de Clamence et de mettre un terme aux polémiques soulevées par le livre. Or, dans les derniers avant-textes du récit, Camus avait désoralisé le monologue de l'avocat et atténué son histrionisme, ouvrant ainsi la porte à d'aisés contresens. Sans être très éloignée du livre qu'il a sous les yeux, mais enrichie des nuances que la voix peut porter mais pas le texte écrit, la version orale de 1958 ôte toute ambiguïté, et, même s'il n'est pas allé jusqu'au bout de son projet (une diffusion radiophonique, par exemple), il est à gager que l'écrivain eût tenu la version

16. Notes manuscrites de Me Henri Barboux pour la défense de Charles et Ferdinand de Lesseps (1893), collection de l'Ordre des avocats de Paris, reproduite ibid., p. 82.

17. En toute hypothèse du moins, puisque de la genèse du discours du 10 décembre 1957 ne subsiste qu'un dactylogramme avec des corrections allographes, dont on peut penser qu'il s'agit d'un document préparatoire à la publication et donc postérieur au discours (voir Euvres complètes, t. IV, op. cit., p. 1398). 
« dite » comme la « bonne » version de La Chute et que le livre même n'eût alors été qu'un avant-dire, ultime dactylogramme préparatoire de la version définitive ${ }^{18}$.

Comme souvent, on doit cependant ici se méfier des faux parallèles : la genèse écrite des genres oraux n'a que bien peu à voir avec la possible genèse orale des genres écrits, même si celle-ci mériterait une analyse qui prît en considération des exemples plus nombreux que le cas de Stendhal dictant en quelques semaines La Chartreuse de Parme à un secrétaire, ou celui de Flaubert soumettant la qualité sonore de sa prose à l'épreuve du « gueuloir ». On pourrait en effet mentionner des cas plus complexes. À deux reprises au moins, Marguerite Duras n'écrivit pas mais enregistra la première version d'un de ses textes, demandant ensuite à un tiers d'opérer une dactylographie de son « avant-texte » et se gardant, dans un dernier temps, de trop intervenir sur le dactylogramme. Elle reprit ainsi en 1980 une première version de l'adaptation théâtrale de la nouvelle de Henry James La Bête dans la jungle, qu'elle avait procurée en 1962 avec James Lord : avec ce premier texte sous les yeux, elle entreprit de « dire » la pièce et reformula - mi-lisant, mi-improvisant - les didascalies et les dialogues, enregistrant sur un magnétophone à cassettes cette " adaptation dite », où elle procède à « une refonte des propos en une écriture lâchée, parlée ${ }^{19}$ ». Elle utilisa la même méthode en 1984, lorsque le metteur en scène Jean-Claude Amyl la sollicita pour une nouvelle version de $L a$ Mouette de Tchekhov20. On notera que, dans les deux cas, il s'agit de « réécritures » de textes dont il convient de s'éloigner, ce qui peut justifier le changement de médium plus encore que le fait qu'il s'agisse de textes théâtraux, dont la première réalisation attendue est orale ; la genèse des pièces personnelles de Duras fut en effet toujours écrite.

$\mathrm{Si}$, par leur rareté même, les cas de genèse orale des textes écrits ne sauraient être mis en parallèle avec les cas, cette fois presque toujours systématiques, de genèse écrite des textes oraux, leur mise en regard n'est pas sans enseignement. Les uns et les autres engagent en effet un imaginaire de la langue. Il est acquis, pour l'historiographie littéraire, que le rapport de l'écrit à l'oral a connu au tournant des XIXe et $\mathrm{XX}^{\mathrm{e}}$ siècle un double mouvement complémentaire : une évidente dévalorisation de l'oral formel (avec le rejet conjoint de la rhétorique et de l'éloquence) ${ }^{21}$ et une réévaluation de l'oral spontané22. Cette dernière fut non seulement marquée par un intérêt vif pour les pratiques populaires, mais aussi par l'idée que le texte écrit devait conserver la sincérité et la saveur prêtées à l'oral non préparé : c'est le fondement d'une entreprise comme Les Confessions de Dan Yack de Blaise Cendrars en 1929 ( « Ce livre n'a pas été écrit. Il a été entièrement dicté au DICTAPHONE. Quel dommage que l'imprimerie ne puisse pas également enregistrer la voix [...] et quel dommage que les

18. J'ai développé cette idée dans « Où commence, où finit la genèse d'une œuvre ? », dans K. Yoshikawa et N. Taguchi (dir.), Comment naît une ouvre littéraire? Brouillons, contextes culturels, évolutions thématiques, Paris, Champion, 2011, p. 329-331.

19. « Le Château de Weatherend », L’Arc, 1983, repris dans Le Monde extérieur (Paris, P.O.L, 1993, p. 119). Les cassettes de ces enregistrements sont aujourd'hui conservées dans les archives de Jean Mascolo, et je remercie Michèle Kastner de m'y avoir donné un très libre accès.

20. Jean-Claude Amyl, dans Théâtre de la Criée/Marcel Maréchal, Approches «Répertoire », $\mathrm{n}^{\circ}$ 19, « Rendez-vous avec le théâtre », août-septembre 1984, présentation de la saison 1984-1985, p. 61-62.

21. Pour un bilan précis et très nuancé de tout ceci, je renvoie à Christelle Reggiani, Éloquence du roman. Rhétorique, littérature et politique aux XIX et XX ${ }^{e}$ siècles, Genève, Droz, 2008.

22. Pour une histoire longue de ceci, je renvoie au chapitre II de Gilles Philippe et Julien Piat (dir.), La Langue littéraire. Une histoire de la prose en France de Gustave Flaubert à Claude Simon, Paris, Fayard, 2009. 
pages d'un livre ne soient pas encore sonores $23 »)$, le fond de la revendication célinienne ou de la fascination contemporaine des écrivains pour la radio ; c'est le début de ce mouvement qui mènera à l'obsession de la « voix » dans une bonne partie de la littérature d'après-guerre. Or, tout cela a configuré notre relation à l'oral préparé et à ses pratiques : si elles sont trop " écrites » les prestations formelles seront disqualifiées voire reçues comme ridicules; elles n'en garderont pas moins le prestige de la belle langue, soutenu par l'idée que la « littérature » s'est désormais réfugiée dans une certaine éloquence. C'est ce double sentiment qui explique la fascination pour les grands discours comme celui par lequel, le 19 décembre 1964, André Malraux accueillit au Panthéon les cendres de Jean Moulin. Pour nous, modernes, le « style » est en effet d'abord une catégorie que nous réservons à l'écrit. Ce mouvement de réduction progressive de l'assiette de la notion, entamé dès la seconde moitié du XIXe siècle mais pas encore tout à fait entériné par le dictionnaire de Littré (« Le langage considéré relativement à ce qu'il a de caractéristique ou de particulier pour la syntaxe et même pour le vocabulaire, dans ce qu'une personne dit, et surtout dans ce qu'elle écrit », s. v. Style), sera complètement accompli dès le début du XXe siècle, avec cependant une hésitation pour l'oral formel préparé. Une note des Cahiers de Paul Valéry témoigne qu'en 1917 celui-ci reste prototypiquement conçu comme un écrit lu et qu'on peut dès lors l'évaluer en termes de style : « Les orateurs ont peu de style. (Je ne parle pas de ceux qui écrivent leurs discours) ${ }^{24}$. » De fait, les spécialistes ont souvent noté que certains tours syntaxiques comme l'apposition, rare dans l'oral spontané, présentaient dans l'oral préparé une densité proche de celle que l'on rencontre à l'écrit, considérant dès lors que conférences ou discours ressortissaient à une catégorie intermédiaire, celle de l'« oral écrit 25 ».

Cette donnée doit être gardée en mémoire, du fait que bien des avant-dire sont aussi des avant-textes et entrent dans un double processus génétique : fort fréquemment en effet, le propos est appelé à être dit mais aussi à être publié. Il devient dès lors délicat de savoir ce qui, dans le document manuscrit, est orienté vers l'horizon oral (avec un allocutaire présent, les conditions propres à un médium «contextualisé » et la possibilité de modifier à tout moment le contenu du propos) et vers l'horizon écrit (avec un allocutaire non identifié, les conditions propres au médium "décontextualisé » et l'impossibilité d'intervenir dans le propos pour l'ajuster, ne serait-ce que par l'inflexion de la voix, aux réactions du public). Pour bien comprendre les enjeux de la question, on pourra partir de ces remarques de JeanMichel Adam :

De façon restreinte, un texte [oral] peut, bien sûr, correspondre à la définition de « discours fixé par l'écriture » que propose Ricœur, mais cela n'est vrai que des discours préparés pour être oralisés (genres du discours politique lu sur un prompteur ou mémorisé, genre de la conférence) ou des transpositions scripturales d'un entretien (dans la presse ou une revue spécialisée) ou d'un discours prononcé. Cependant, dans ces deux derniers cas, la transposition scripturale ne garde pas la rythmicité, les pauses, les intonations et les hésitations significatives de l'oral. Les discours prononcés par de Gaulle, Malraux, Chirac, Obama ou Sarkozy diffèrent parfois énormément de leur(s) version(s) écrite(s). Il en va de même d'une conférence préparée scripturalement (état T1),

23. Blaise Cendrars, Les Confessions de Dan Yack (1929), Euvres complètes, t. III, Paris, Denoël, 1960-1965, p. 128. 24. Paul Valéry, Cahiers, t. I, éd. J. Robinson-Valéry, Paris, Gallimard, coll. « Bibliothèque de la Pléiade », 1989, p. 403.

25. Voir Joëlle Gardes Tamine, Pour une grammaire de l'écrit, Paris, Belin, 2004, p. 151. 
prononcée ensuite (état T2) et enfin publiée dans un volume (état T3). Les régimes oral et scriptural sont des régimes de textualité dont les contraintes diffèrent $[\ldots]^{26}$.

Tout cela permet de rendre compte de la difficulté de l'analyse génétique des avant-dire, dès lors que ceux-ci sont souvent aussi des avant-textes au sens étroit du terme : l'état T1 participe de la genèse de l'état T2 mais aussi de l'état T3. Cette double finalité est alors gérée simultanément : le texte préparé doit pouvoir satisfaire aux exigences de l'oralisation (T2) mais aussi à celles de la publication (T3). L'état T3 a vocation à être différent de l'état T2, puisque les contraintes de l'énonciation écrite diffèrent de celles de l'énonciation orale, mais il ne doit pas l'effacer : sous peine de sortir du genre qui lui est assigné et de perdre donc une grande partie de sa saveur, le discours publié ne doit pas faire oublier qu'il a été prononcé. Il doit se donner, au moins fictivement ou contractuellement, comme la transposition voire la transcription du discours prononcé. Même quand l'état T3 est une version complètement retravaillée de l'état T2, il en garde généralement la scénographie minimale, c'est-à-dire les formes de l'adresse.

On illustrera ici la question, plus qu'on ne l'étudiera, à partir d'un cas simple, volontairement emprunté à Gustave Lanson que l'histoire a retenu comme un pourfendeur de la rhétorique. Il s'agit d'un extrait de la transcription d'une allocution donnée en 1917 et corrigée de la main de l'auteur en vue d'une publication. Le dactylogramme (fig. 3) semble être la saisie par un tiers du discours tel qu'il a été prononcé (on note l'indication des «Applaudissements ») ou plutôt entendu (noms propres et références sont fréquemment estropiés) ${ }^{27}$.

\section{Je ne sais pas si vos livres sont eomme eela, et alors $j<J>e$}

me demandais s'il ne serait pas possible de trouver, dans nos grands <et $>$ éerivains, nos bons écrivains, des phrases qui diraient quelque chose $<,>$ et qui $<$ pourraient $>$ serviraient d'exemple $<$ s $>$ : parce qu'elles indiqu $<$ signifi $>$ eraient quelque chose, elles graveraient <resteraient> d'autant mieux dans l'esprit de l'élève,-<.> $\mathrm{b}<\mathrm{B}>$ ]ien que $\mathrm{j}<\mathrm{J}>\mathrm{e}$ sois $<$ suis $>$, en principe, pour les commençants, de l'avis qu'il vaut mieux employer $<$ faire étudier de $>$ la prose que $1<\mathrm{d}>$ es vers, mais $<$ je ferais [valoir] $>$ il n'y a rien qui se grave mieux $<$ dans l'esprit $>$ qu'un vers, et un $<$ on pourrait choisir des $>$ exemple $<$ s $>$ grammaticat $<$ aux $>$ tirré d'un vers $<$ dans notre poésie $>$;. et pour les rè $<$ Rien ne serait plus aisé que d'illustrer les rè $>$ gles $\mathrm{de}<\mathrm{s}>$ participe $<\mathrm{s}>$, on en prend dans les vers $<$ par les vers de nos grands poètes $>$. <On pourrait choisir des [segment non utilisé]. Et puis, voità $<$ Voici $>$ un vers de Victor Hugo qui me revient <à la mémoire $>$

« Près des motlins <meules $>$ ete... < qu'on eût prises pour des décombres. $>$

Voilà l'accord $<$ : $>$ on voit dans quel cas il se fait $<$; $>$ et l'élève ne pourra $<$ it $>$ plus $<$ pas $>$ dire : «Près des moulins $<$ meules $>$ ete... < qu'on eût pris pour des décombres.>

$<$ Le vers serait faux. $>$ La $<$ Ailleurs la $>$ règle lui sera rappelée eomme $<$ par $>$ la rime. Cela $<$ Ce moyen $>$ peut se faire $<$ s'employer $>$ d'une façon courante.

26. Jean-Michel Adam, «Introduction aux problèmes du texte », dans J.-M. Adam (dir.), Faire texte. Unité(s) et (dis)continuité, Besançon, Presses de l'Université de Franche-Comté, à paraître.

27. Je note entre soufflets les ajouts manuscrits autographes (le plus souvent interlinéaires) ; entre crochets sont données les lectures conjecturales. Je remercie vivement la petite-fille de Gustave Lanson, Mme Denise Blanc, de m'avoir donné accès à ce document. 
Il est clair que cet état proche de T2 ne saurait être celui de T1 (que nous ne possédons plus) : même si Lanson a prononcé un texte rédigé, ce qui est probable, il est peu vraisemblable que celui-ci ait contenu l'alignement sur le standard oral (« Et puis voilà », par exemple) que manifeste notre T2'. On notera que le retravail en vue de T3, atténue les faits de connivence (biffure de «Je ne sais pas si vos livres sont comme cela », par exemple) et marque une certaine élévation du niveau lexical. Pour autant, le texte est, à ce stade d'élaboration de T3, appelé à garder son allure un peu informelle ; sa rédaction reste simple, le ton en demeure personnel. Pour des raisons que nous ignorons, Lanson n'a pas souhaité ou pas pu travailler à partir de $\mathrm{T} 1$, mais il est surtout certain qu'il a voulu garder à sa prose quelque chose d'oral et conserver les conventions génériques de l'allocution ${ }^{28}$.

J'ai voulu ici privilégier l'exemple sur la théorie. Il importe en effet de prendre d'abord acte de la complexité non seulement de la matérialité de l'avant-dire mais aussi des configurations génétiques dans lesquelles il entre. Si l'écrire pour écrire ne connaît pas ou guère les entrelacs de l'écrire pour parler, c'est que la continuité du médium simplifie la perception du mouvement téléologique qui mène des premières notes au texte final. En soi, en effet, la présentation graphique de l'avant-dire ne diffère guère de celle des avant-textes dont nous sommes plus familiers. Mais là n'est pas l'essentiel, nous l'avons vu, et comme seule la comparaison permet de faire apparaître le spécifique, gageons que la génétique des textes écrits elle-même a tout à gagner de l'étude des entrelacs génétiques dans lesquels sont pris les avant-dire.

28. Il est vrai que le texte était appelé à une diffusion très limitée : il a paru sous le titre « La fonction du professeur français à l'étranger », dans le compte rendu du Troisième Congrès de langue et de littérature françaises (Chicago, 25-26 mai 1917), diffusé par le secrétariat de la Fédération de l'Alliance française, New York). Je dois toute reconnaissance à Martine Jey qui a pu reconstituer l'histoire de ce texte mineur. 


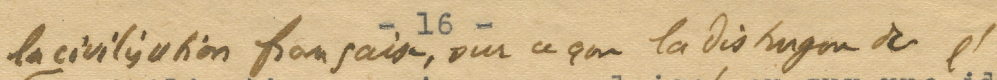
chose sur/ la civilisation romaine, ou anglaise/ ou, sur lidee generacivilisation las and órcoiñ;

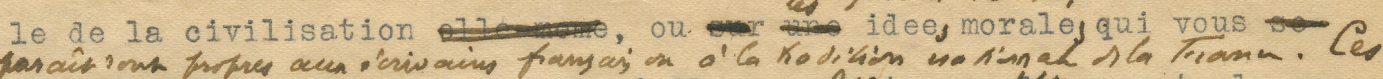

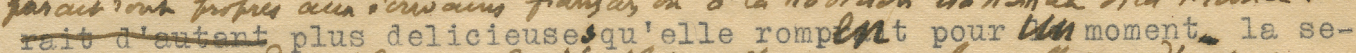

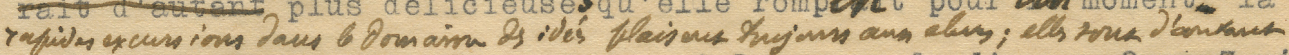
verite de I'exercice pedagogique, etjle moyen de dire en eing minutes un tas de choses, (tout on le programmestrous pouvons

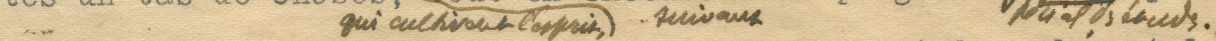
quelque chose de plus oncore: je me suis souvent demande- - et $1 \dot{a}$, je ne parle pas avec une connaissance hien precise, des livres dont vous

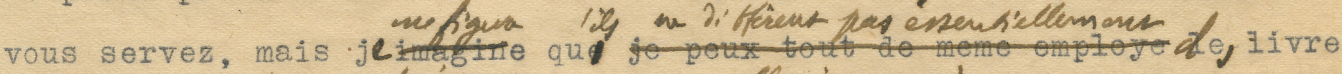

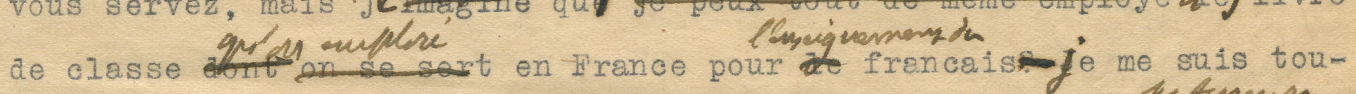
jours demandé quelle etait la necessité qui obligeait les fufrows tors

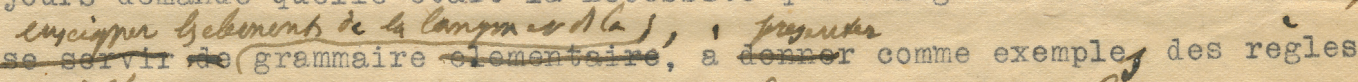

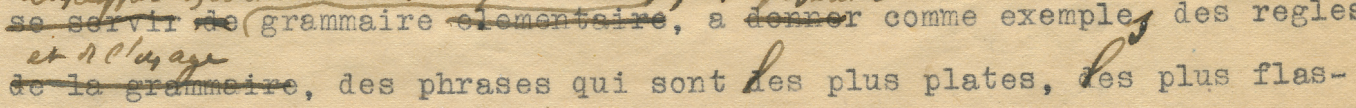

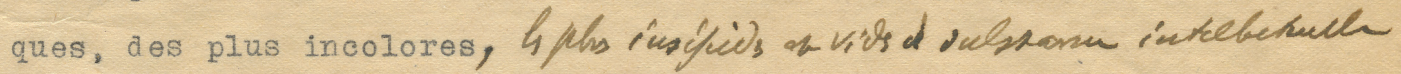
qion puim icnogine. (Applaudissements)

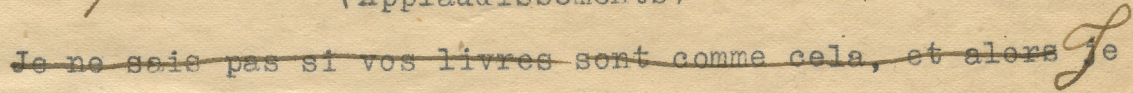

me demandais s'il ne serait pas possible de trouver, dans nos grands eh hos bons ecrivains, des phrases qui diraient quelque chose, et qui servir a'exemple, parce qu'elles opificieraient quelque hose, elles reftriem d'autant mieux dans l'esprit ae l'eleve. Jo mis, en principe, pour les commengants, de l'avis qu'il vaut mieux prose que des vers, mais il n'y a rien qyi se grave mieux

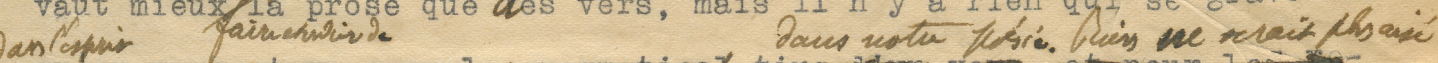
qu un vers, et exemplejgramaticata cies des partio fipesain clenisis oy

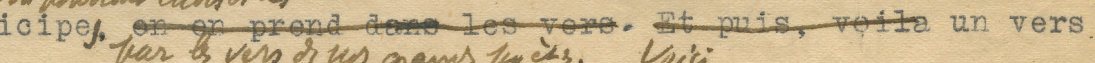

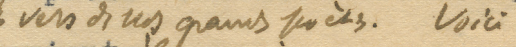

de Victor Hugo qui me revienta'la inemain

"Pres des mluiles otion eñ fripe fous \& drombs.

Voile I'accord: on voit dans quel cas il se fait; et l'eleve

ne pourra; fles aire: 6 ven mair "Pres

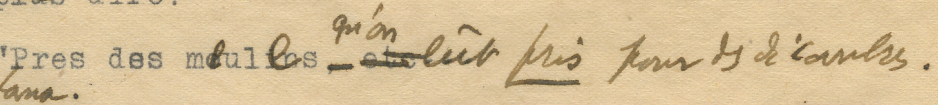

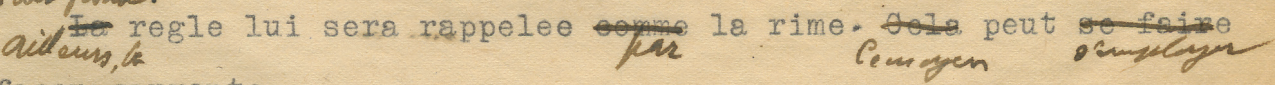
d'une facon courante.

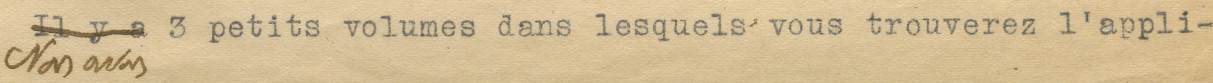

Fig. 3 : Gustave Lanson, dactylogramme corrigé (coll. particulière) 
Gilles Philippe est professeur de linguistique française à l'Université de Lausanne. Ses travaux portent sur les imaginaires langagiers et les pratiques stylistiques aux XIX et Xxe siècles. Il est notamment l'auteur de Le Français, dernière des langues. Histoire d'un procès littéraire (Presses universitaires de France, 2010) et Le Rêve du style parfait (Presses universitaires de France, 2013). Il a procuré de nombreuses éditions génétiques pour la «Bibliothèque de la Pléiade » (Gallimard).

gilles.philippe@unil.ch

\section{Écrire pour parler. Quelques problématiques premières}

Si le texte écrit pour être dit embarrasse, c'est qu'un document fait toujours défaut : celui de la parole vive, saisie dans l'instant même, et qui est l'état « final » à l'aune duquel l'avant-dire devrait être considéré. Ce manque ne doit cependant pas arrêter toute réflexion, et l'on essaie ici de recenser les principales questions qui se posent. Le brouillon de l'oral diffère-t-il matériellement et linguistiquement du brouillon de l'écrit ? Jusqu'à quel point anticipe-t-il la prestation orale et de quelle façon? Son statut pragmatique est-il le même que celui d'un avant-texte ordinaire ? Est-il symétrique au cas inverse $\mathrm{du}$ « brouillon oral » d'un texte écrit? Comment s'articule-t-il aux évolutions des imaginaires sociaux de l'écrit et de l'oral ? Comment gère-t-il sa possible double finalité, dès lors qu'une conférence ou un discours, par exemple, sont aussi appelés à être publiés, et que l'avant-dire est aussi un avant-texte au sens habituel du terme?

If a text written to be said is troublesome, it is because a document is always missing: that of the spoken word, seized in the very instant, which is the "final" state in the light of which the "avantdire" should be considered. However, this deficiency should not put a stop to all thinking. We will try here to list the main issues that arise. Does the draft of an oral text differ materially and linguistically from the written draft? Up to what point does it anticipate the oral performance and in what way? Is its pragmatic status the same as that of an ordinary "avant-texte"? Is it symmetrical to the opposite case of a written text's "oral draft"? How is it articulated to the evolutions of the social imaginaries of the written and the oral? How does it manage its possible double purpose, when a lecture or a speech, for example, are also meant to be published, and when the "avant-dire" is also an "avant-texte" in the usual sense of the term?

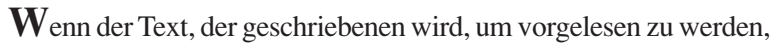
störend erscheint, dann, weil stets etwas fehlt: das lebendige Wort, das im selben Moment wahrgenommen wird und den „,finalen“ Zustand darstellt, an dessen Maßstab das „Avant-Dire“ "betrachtet werden sollte. Dieser Mangel darf jedoch nicht die gesamten Überlegungen stoppen; die wichtigsten Fragen, die sich stellen, sollen hier erfasst werden. Unterscheidet sich der Entwurf des Mündlichen materiell und sprachlich vom Entwurf des Schriftlichen? Bis zu welchem Grad und auf welche Weise antizipiert er die mündliche Darbietung? Ist sein pragmatischer Status der gleiche wie jener eines gewöhnlichen Avant-Texts? Ist er symmetrisch zum umgekehrten Fall des „mündlichen Entwurfs“ eines schriftlichen Texts? Wie knüpft er an die Entwicklungen bezüglich der gesellschaftlichen Vorstellungen des Schriftlichen und des Mündlichen an? Wie geht er mit der möglicherweise zweifachen Finalität um, wenn beispielsweise ein Vortrag oder eine Rede auch publiziert werden soll und das Avant-Dire auch einen Avant-Text im eigentlichen Sinne des Begriffes darstellt?
$\mathbf{S}_{\mathrm{i}}$ un texto escrito para ser dicho perturba, es porque en general falta un documento: el de la palabra viva, captada en el instante mismo y que constituye el estado "final" en función del cual el predecir tendría que ser considerado. Sin embargo, como esa ausencia no debe impedir la reflexión, trataremos aquí de identificar las principales cuestiones que se plantean. ¿El borrador de una alocución es diferente, material y lingüísticamente, del borrador de un texto escrito? ¿Hasta qué punto anticipa la manifestación oral y de qué manera lo hace? ¿Tiene el mismo nivel pragmático que el de un pre-texto ordinario? ¿Es simétrico con respecto al caso inverso de un "borrador oral" de un texto escrito? ¿Cómo se articula con las evoluciones de los imaginarios sociales de lo escrito y de lo oral? ¿Cómo se adapta a su posible doble finalidad, teniendo en cuenta que una conferencia o un discurso, por ejemplo, pueden estar destinados a ser publicados y que en este caso el pre-decir es también un pre-texto en el sentido habitual del término?

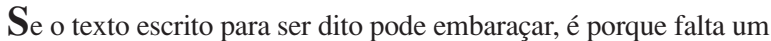
documento: o da palavra viva, captada no momento e constituindo o estado "final" em relação ao qual o ante-dito deveria ser considerado. No entanto, esta falta não suspender a reflexão, pelo que aqui serão recenseadas as principais questões que se colocam. O rascunho do oral difere material e linguisticamente do rascunho da escrita? Até que ponto e de que medida condiciona ele a prestação oral? Cabe-lhe estatuto pragmático idêntico ao de um vulgar antetexto? Ou, na inversa, é simétrico do "rascunho oral" de um texto escrito? Como se articula com a evolução do imaginário social do escrito e do oral? Como ele gere ele a possibilidade de ter uma dupla finalidade, na medida em que uma conferência ou um discurso, por exemplo, podem vir a ser publicados, e que o ante-dito é também um ante-texto no sentido habitual do termo?

S e il testo scritto per essere pronunciato pone dei problemi è a causa della mancanza inevitabile di un documento: quello della parola viva, colta nell'istante stesso, che ne rappresenta lo stadio "finale" al confronto del quale l'avandetto dovrebbe essere considerato. Questa mancanza non deve tuttavia impedire la riflessione; tenteremo perciò di affrontare in quest'articolo le principali domande che sorgono. La prima stesura dell'orale è materialmente e linguisticamente diversa da quella dello scritto? Fino a che punto anticipa la prestazione orale e in che modo? Ha lo stesso statuto pragmatico di un normale avantesto? È simmetrica al caso opposto della "prima stesura orale" di un testo scritto? Come si articola rispetto alle evoluzioni degli immaginari sociali dello scritto e dell'orale? In che modo gestisce la sua possibile doppia finalità quando, ad esempio, un discorso o una conferenza sono destinati alla pubblicazione, e di conseguenza l'avandetto è anche un avantesto nel senso usuale del termine? 